\title{
IMPACTO DE LA AGRICULTURA Y GANADERÍA SOBRE EL BOSQUE TROPICAL SECO DE ZIRÁNDARO, GUERRERO: UNA EVALUACIÓN CON INDICADORES ECOLÓGICOS
}

\section{IMPACT OF AGRICULTURE AND CATTLE RAISING ON THE TROPICAL DRY FOREST OF ZIRÁNDARO, GUERRERO: AN EVALUATION USING ECOLOGICAL INDICATORS}

\author{
Agustín Fuentes-Hernández, Marleth Mendoza-Orozco, leticia Ríos-Casanova, Alfonso Soler-Aburto, \\ Daniel Muñoz-INIESTRA y HÉCTOR Godínez-Álvarez*
}

Unidad de Biología, Tecnología y Prototipos, Facultad de Estudios Superiores Iztacala, Universidad Nacional Autónoma de México, Tlalnepantla, Estado de México, México

*Autor de correspondencia: hgodinez@unam.mx

\begin{abstract}
Resumen
Antecedentes: La agricultura y ganadería modifican la estructura y funcionamiento del bosque tropical seco. El impacto de estas actividades ha sido evaluado en regiones de Jalisco y Yucatán, en donde la agricultura de roza, tumba y quema, y la ganadería extensiva son los principales tipos de manejo. Sin embargo, existen otras regiones como Guerrero, en donde la agricultura es combinada con la ganadería. No obstante, su impacto sobre el bosque no ha sido evaluado hasta el momento.

Pregunta: ¿Cuál es el impacto de la agricultura/ganadería y ganadería sobre el bosque tropical seco de Zirándaro, Guerrero?

Sitio y años de estudio: El estudio fue realizado en Zirándaro, Guerrero, en diciembre de 2014 y junio de 2015.

Métodos: Se seleccionaron 9 sitios con agricultura/ganadería, ganadería y bosque para muestrear su vegetación y suelo, y calcular 10 indicadores ecológicos. Además, se colectaron 9 muestras de suelo para evaluar sus propiedades físicas y químicas.

Resultados: Los suelos de la agricultura/ganadería y ganadería tuvieron mayor densidad aparente, pero menor porosidad y materia orgánica que el suelo del bosque. La agricultura/ganadería y ganadería tuvieron baja riqueza específica y cobertura del dosel. La agricultura/ganadería tuvo menor obstrucción visual y mayor proporción de claros basales que la ganadería. Estas diferencias sugieren que la agricultura/ganadería tiene mayor impacto sobre el bosque que la ganadería. Sin embargo, no se encontraron diferencias significativas en la mayoría de los indicadores ecológicos.
\end{abstract}

Conclusiones: La agricultura/ganadería y ganadería tienen un impacto ecológico relativamente similar sobre el bosque tropical seco de Zirándaro, Guerrero.

Palabras clave: Agricultura de temporal, Cuenca del Balsas, degradación de la tierra, ganadería extensiva, selva baja caducifolia.

\begin{abstract}
Background: Agriculture and cattle raising modify the structure and functioning of tropical dry forest. The impact of these activities has been evaluated in regions of Jalisco and Yucatán, where slash-and-burn agriculture and extensive cattle raising are the main types of management. However, there are other regions such as Guerrero, where agriculture is combined with cattle raising. Yet, its impact on the forest has not been evaluated so far.

Question: What is the impact of agriculture/cattle raising and cattle raising on the tropical dry forest in Zirándaro, Guerrero?

Study sites and dates: The study was performed in Zirándaro, Guerrero, in December 2014 and June 2015.

Methods: We selected 9 sites with agriculture/cattle raising, cattle raising, and forest to sample vegetation and soil, and to calculate 10 ecological indicators. We also collected 9 soil samples to evaluate their physical and chemical properties.

Results: The soil of agriculture/cattle raising and cattle raising had higher bulk density, but lower porosity and organic matter content than the forest soil. The agriculture/cattle raising and cattle raising had low plant species richness and canopy cover. The agriculture/cattle raising had lower vegetation structure and higher proportion of basal gaps than cattle raising. These differences suggest that agriculture/cattle raising had a higher impact on forest than cattle raising. Yet, we did not find significant differences in most of the ecological indicators.

Conclusions: The agriculture/cattle raising and cattle raising have a relatively similar ecological impact on the tropical dry forest of Zirándaro, Guerrero.

Key words: Balsas Basin, extensive cattle raising, land degradation, rainfed agriculture, tropical deciduous forest.
\end{abstract}

This is an open access article distributed under the terms of the Creative Commons Attribution License CCBY-NC (4.0) international. https://creativecommons.org/licences/by-nc/4.0/ 
La agricultura y ganadería modifican la estructura y funcionamiento de los bosques (Challenger 1998). Los campos agrícolas y pastizales ganaderos son ecosistemas con baja riqueza, abundancia y diversidad de plantas. Además, tienen una vegetación simple que favorece el incremento de la temperatura del suelo y el aire, y disminuye la humedad del suelo (Arriaga 2006, Morales-Romero \& Molina-Freaner 2016). Estos cambios disminuyen la capacidad del suelo para almacenar agua, incrementan la pérdida de nutrientes por erosión y reducen la productividad primaria (Arriaga 2006, García-Oliva et al. 2006a, 2006b). Todas las modificaciones causadas por la agricultura y ganadería disminuyen la regeneración de los bosques y su capacidad de recuperación (Hernández-Vargas et al. 2000, Arriaga 2006), por lo que es necesario evaluar el impacto ecológico de estas actividades.

Una alternativa para evaluar el impacto de la agricultura y ganadería sobre los bosques son los indicadores ecológicos. Estos indicadores son mediciones relativamente fáciles y rápidas de los componentes físicos y biológicos de los ecosistemas que proporcionan información sobre su estructura y funcionamiento (Pellant et al. 2005). Así, por ejemplo, mediciones como la cobertura del dosel, la proporción de claros basales y la compactación del suelo proporcionan información sobre la capacidad de un área para limitar la redistribución y pérdida de nutrientes y materia orgánica del suelo (i.e., la estabilidad del sitio/suelo; Pyke et al. 2002, Herrick et al. 2009b, 2017). Otras mediciones como la cobertura del suelo desnudo, la cobertura de la hojarasca y la infiltración proporcionan información sobre la captura, almacenamiento y distribución de la lluvia y escorrentía superficial (i.e., la función hidrológica; Pyke et al. 2002, Herrick et al. 2009b, 2017). Por último, mediciones como la riqueza específica de plantas, la cobertura de la hojarasca y la obstrucción visual proporcionan información sobre la capacidad de un área para sostener el flujo de energía y el ciclo de los nutrientes dentro del rango normal de variabilidad del sitio (i.e., la integridad biótica; Pyke et al. 2002, Herrick et al. 2009b, 2017). Debido a que los indicadores proporcionan datos sobre la estructura y funcionamiento de un ecosistema, pueden usarse para comparar sitios con distinto tipo de manejo que estén ubicados en la misma unidad del paisaje, en donde la vegetación y el tipo de suelo son relativamente similares. En general, los sitios con manejo inadecuado tendrían pocas especies de plantas, baja cobertura de dosel, hojarasca y obstrucción visual, alta proporción de claros, alta cobertura de suelo desnudo y compactación, y baja infiltración, en comparación con los sitios con manejo adecuado.

En México, el impacto de la agricultura y ganadería sobre el bosque tropical seco ha sido estudiado en la región costera de Jalisco y en las Penínsulas de Yucatán y Baja California (Challenger 1998, Arriaga 2006, García-Oliva et al. 2006a). En estas regiones, la agricultura de roza, tumba y quema, y la ganadería extensiva son las principales actividades agropecuarias que impactan el bosque. En general, estas actividades afectan negativamente la estructura de las comunidades de plantas, las propiedades físicas y químicas del suelo y la dinámica de algunos nutrientes como el carbono, nitrógeno y fósforo (García-Oliva et al. 1994, 2006b, Miller 1999, Giardina et al. 2000, Kauffman et al. 2003, Martínez-Trinidad et al. 2008, Sandoval-Pérez et al. 2009). Aunque esta información es significativa, los estudios sobre el impacto de las actividades agropecuarias en otras regiones de bosque tropical seco de nuestro país como la Cuenca del Balsas, en Guerrero y Oaxaca, son limitados (Meave et al. 2012). El bosque tropical seco en la región de Zirándaro, Guerrero, ha sido transformado a campos de cultivo y pastizales ganaderos. Sin embargo, el manejo en esta región difiere del manejo en Jalisco y Yucatán, debido a que los agricultores no queman las plantas después de la roza e introducen ganado bovino después de la cosecha. Además, la transformación del bosque ha ocurrido en las laderas de los cerros, por lo que el riesgo de erosión del suelo y la pérdida de nutrientes son altos (M. Mendoza-Orozco, observación personal). La combinación de agricultura/ganadería en esta región de Guerrero podría tener un impacto ecológico distinto al impacto de la agricultura de roza, tumba y quema en Jalisco y Yucatán. Sin embargo, hasta el momento no existe ninguna evaluación del impacto de estas actividades agropecuarias en esta región.

En este estudio se evalúa el impacto de la agricultura/ ganadería y ganadería sobre la estructura y funcionamiento del bosque tropical seco de Zirándaro, Guerrero. Para esto, se seleccionaron sitios con agricultura/ganadería, ganadería y bosque, en donde se midieron indicadores ecológicos (riqueza específica, cobertura de dosel, hojarasca y suelo desnudo, proporción de claros, obstrucción visual, estabilidad y compactación del suelo, e infiltración) y se evaluaron propiedades del suelo (textura, densidad, materia orgánica y $\mathrm{pH})$. Se espera que los sitios con agricultura/ganadería y ganadería tengan menor estructura y funcionamiento que los sitios con bosque.

\section{Materiales y métodos}

Zona de estudio. El estudio fue realizado en Zirándaro, Guerrero, México ( $18^{\circ} 32^{\prime} 17^{\prime \prime} \mathrm{N}, 100^{\circ} 54^{\prime} 53^{\prime \prime} \mathrm{O}, 220 \mathrm{~m}$ s.n.m.). El clima es seco cálido y la temperatura promedio anual es $29{ }^{\circ} \mathrm{C}$ (García 2004). La precipitación promedio anual es $400 \mathrm{~mm}$ y está concentrada entre julio y octubre. Los suelos son Litosol, Feozem y Regosol (INEGI 2001). La vegetación original es el bosque tropical seco dominado por Bursera excelsa, Caesalpinia coriaria, Conzattia multiflora, Lysiloma tergemina, Ziziphus amole y Cordia elaeagnoides (M. Mendoza-Orozco, datos no publicados). Sin embargo, el bosque ha sido transformado a campos de agricultura de riego en las partes bajas del valle y a campos de agricultura de temporal o pastizales ganaderos en las laderas de los cerros. Aunque todavía existen remanentes de bosque en las laderas de los cerros.

Para evaluar el impacto del manejo se seleccionaron tres sitios con agricultura/ganadería, ganadería y bosque, para un total de nueve sitios. Se seleccionaron tres sitios para considerar la variación de cada tipo de manejo. Los sitios con agricultura/ganadería tienen una superficie de 2-5 ha. La vegetación está conformada por algunos árboles en pie 
como Haematoxylum brasiletto y C. elaeagnoides. El manejo consiste en cultivar maíz y sorgo de temporal de julio a octubre y mantener 10 cabezas/ha de ganado bovino de las razas Suizo y Brahman de noviembre a mayo, por lo que tienen un manejo combinado. Los sitios con ganadería tienen una superficie de 2-5 ha. La vegetación está conformada por el pasto Andropogon gayanus y algunos árboles en pie como Crescentia alata y Caesalpinia coriaria. El manejo consiste en mantener en promedio 6 cabezas/ha de ganado bovino de las razas Suizo y Brahman de julio a septiembre y dejar descansar la tierra de octubre a junio. Los sitios con bosque tienen una superficie de 2-17 ha. La vegetación está conformada por árboles y arbustos. El único manejo consiste en extraer productos forestales no maderables como ramas secas para leña y plantas medicinales para uso doméstico, 1-2 veces por año (M. Mendoza-Orozco, datos no publicados). La distancia entre los sitios con agricultura/ganadería, ganadería y bosque fue de 500-1931 m. Los nueve sitios fueron ubicados en laderas de cerros (altitud: 223-302 m s.n.m., orientación: NE, E y SO, pendiente: 15-25\%) para que la unidad geomorfológica, la vegetación y el tipo de suelo fueran relativamente similares y sólo difirieran en el tipo de manejo.

Indicadores ecológicos. En cada sitio, se realizaron seis mediciones de la vegetación y el suelo y, con los datos obtenidos se calcularon 10 indicadores. Las seis mediciones de la vegetación y el suelo fueron: (1) el intercepto líneapunto, (2) el intercepto de claros, (3) la estructura de la vegetación, (4) la estabilidad del suelo, (5) la infiltración y (6) la compactación del suelo. Para cada una de estas mediciones se colocaron, en la parte central de cada sitio, tres líneas paralelas de $30 \mathrm{~m}$ de longitud sobre la superficie del suelo, separadas $10 \mathrm{~m}$ cada una. La colocación de las líneas y su longitud permitieron representar las condiciones de suelo y vegetación predominantes en cada sitio. Para el intercepto línea-punto (Herrick et al. 2009a), se colocó una pértiga de $3 \mathrm{~m}$ de alto, cada metro sobre la línea, para registrar una sola vez las especies de plantas, hojarasca, rocas y suelo desnudo que eran interceptados por la pértiga. Con los puntos interceptados por las especies de plantas, hojarasca, rocas y suelo desnudo, y el total de puntos por línea se calcularon los indicadores (escala): (1) riqueza específica de plantas (número de especies/línea), (2) cobertura del dosel (proporción), (3) cobertura de hojarasca (proporción) y (4) cobertura de suelo desnudo (proporción). Para el intercepto de claros (Herrick et al. 2009a), se registró el inicio y término de los claros en el dosel y los claros basales que eran interceptados por la línea. Ambos claros, dosel y basales, fueron clasificados en cuatro categorías dependiendo de su longitud $(50-100 \mathrm{~cm}, 101-500$ $\mathrm{cm}, 501-1,000 \mathrm{~cm},>1,000 \mathrm{~cm})$. Con la suma de la longitud por categoría y la longitud total de la línea se calcularon los indicadores: (5) claros en el dosel de 50-100 cm, 101-500 $\mathrm{cm}, 501-1,000 \mathrm{~cm} \mathrm{y}>1,000 \mathrm{~cm}$ (proporción) y (6) claros basales de 50-100 cm, 101-500 cm, 501-1,000 cm y $>1,000$ $\mathrm{cm}$ (proporción). Para la estructura de la vegetación (Herrick et al. 2009b), se colocó una pértiga de $3 \mathrm{~m}$ de alto, dividida en 30 segmentos de $10 \mathrm{~cm}$, a los 5, 15 y $25 \mathrm{~m}$ de la línea, para contar el número de segmentos cubiertos por la vegetación. Los segmentos fueron observados a través de un tubo de PVC perforado a una altura de $140 \mathrm{~cm}$, que fue colocado 5 $\mathrm{m}$ antes y después de la pértiga. Con el número de segmentos cubiertos por la vegetación y el número total de segmentos se calculó el indicador: (7) obstrucción visual (proporción). Para la estabilidad del suelo (Herrick et al. 2009a), se colectó una muestra de agregados del suelo de 6-8 $\mathrm{mm}$ de ancho y 2-3 mm de alto, cada $10 \mathrm{~m}$ sobre la línea, para determinar su estabilidad después de sumergirla en agua destilada por 5 min. La estabilidad fue determinada con una escala de 1-7, en donde 1 es poco estable y 7 es muy estable, de acuerdo con el método propuesto por Herrick et al. (2009a). Con estos datos se estimó el indicador: (8) estabilidad del suelo (1-7). Para la infiltración, se enterró un tubo de PVC $(10 \mathrm{~cm}$ de diámetro por $30 \mathrm{~cm}$ de longitud) a $3 \mathrm{~cm}$ de profundidad, cada $10 \mathrm{~m}$ sobre la línea, para medir el tiempo de infiltración de 250 $\mathrm{ml}$ de agua. Esta profundidad permitió medir la infiltración en suelos poco alterados por la instalación del tubo de PVC. Sin embargo, esta medición podría estar sesgada por el flujo lateral del agua. Con estos datos se estimó el indicador: (9) infiltración (min). Para la compactación, se colocó un penetrómetro estático con manómetro, cada $10 \mathrm{~m}$ sobre la línea, para medir la presión necesaria para enterrarlo a $2.5 \mathrm{~cm}$ de profundidad. Con estos datos se estimó el indicador: (10) compactación $\left(\mathrm{kg} / \mathrm{cm}^{2}\right)$. Los muestreos de suelo y vegetación fueron realizados en diciembre de 2014 y junio de 2015, durante la temporada de sequía.

Características del suelo. Además de los métodos para la vegetación y suelo, se colectó una muestra de $500 \mathrm{~g}$ de suelo en los primeros $10 \mathrm{~cm}$ de profundidad a los $15 \mathrm{~m}$ de cada línea, para evaluar sus propiedades físicas y químicas. Las muestras fueron mezcladas para obtener una muestra compuesta por sitio, para un total de 9 muestras compuestas. Con estas muestras compuestas se evaluó: (1) textura con el método volumétrico, (2) densidad aparente con el método de la probeta, (3) densidad real con el método del picnómetro, (4) materia orgánica con el método de oxidación con ácido crómico y sulfúrico, y (5) $\mathrm{pH}$ con potenciómetro digital y solución suelo-agua 1:2.5 (Muñoz et al. 2013). Las muestras de suelo fueron tomadas en diciembre de 2014, durante la temporada de sequía.

Análisis estadístico. Los indicadores ecológicos y las propiedades del suelo fueron comparadas entre los sitios con agricultura/ganadería, ganadería y bosque con análisis de varianza de una vía y comparaciones múltiples de Tukey-Kramer, excepto los claros en el dosel y los claros basales. Estos últimos fueron analizados con análisis de varianza anidados, en los cuales los claros fueron anidados dentro de los sitios. Los indicadores y las propiedades expresadas en proporción o porcentaje fueron transformadas con la función arcoseno, mientras que las expresadas como conteos fueron transformadas con el logaritmo decimal $(\mathrm{x}+1)$, para cumplir los supuestos de normalidad e igualdad de varianzas de las pruebas estadísticas. Todos los análisis estadísticos fueron realizados con el programa JMP versión 10 (SAS 2012). 


\section{Resultados}

Características del suelo. El suelo tuvo porcentajes similares de arena, limo y arcilla en los tres tipos de manejo (Tabla 1). Sin embargo, los suelos de la agricultura/ganadería y ganadería tuvieron mayor densidad aparente $\left(F_{2,24}=30.8\right.$, $p<0.0001)$ y $\mathrm{pH}\left(F_{2,24}=20.5, p<0.0001\right)$, así como menor porosidad $\left(F_{2,24}=10.1, p=0.0007\right)$ y materia orgánica $\left(F_{2,24}=5.3, p<0.013\right)$ que el suelo del bosque.

Indicadores ecológicos. La riqueza específica de plantas $\left(F_{2,4}=25.9, p<0.0001\right)$ y la cobertura del dosel $\left(F_{2,24} \stackrel{2,24}{=} 31.4, p<0.0001\right)$ en la agricultura/ganadería y ganadería fueron similares, pero menores que en el bosque (Figura 1A-B). Con respecto a la obstrucción visual $\left(F_{2,159}=66.9, p<0.0001\right)$, la agricultura/ganadería tuvo el menor valor seguida por la ganadería y el bosque (Figura 1C). La cobertura de la hojarasca fue similar en los tres tipos de manejo $\left(F_{224}=0.8, p=0.5\right.$; Figura 1D). La proporción de claros en el dosel $>1,000 \mathrm{~cm}$ fue baja en la agricultura/ganadería, mientras que en la ganadería fue alta (Sitio: $F_{1,64}=0.4, p=0.5$; Claro[sitio]: $F_{6,64}=9.5, p<0.0001$ ). Por el contrario, la proporción de claros basales $>1,000 \mathrm{~cm}$ fue alta en la agricultura/ganadería, mientras que en la ganadería fue baja (Sitio: $F_{1,64}=0.0004, p=0.9$; Claro[sitio]: $F_{6,64}=6.6$,
Tabla 1. Propiedades del suelo (media \pm error estándar) en sitios con distinto manejo. Para cada propiedad, las letras señalan diferencias significativas en el manejo $(p<0.05)$.

\begin{tabular}{lccc}
\hline & \multicolumn{3}{c}{ Manejo } \\
\cline { 2 - 4 } & $\begin{array}{c}\text { Agricultura/ } \\
\text { Ganadería }\end{array}$ & Ganadería & Bosque \\
\hline Arena (\%) & $70.7 \pm 1.8$ & $64.7 \pm 3.7$ & $64.7 \pm 1.3$ \\
Limo (\%) & $23.3 \pm 2.3$ & $24.0 \pm 1.2$ & $28.0 \pm 3.5$ \\
Arcilla (\%) & $6.0 \pm 1.5$ & $11.3 \pm 2.9$ & $7.3 \pm 2.2$ \\
$\begin{array}{l}\text { Densidad aparente } \\
\text { (kg/m } \text { ( }^{3}\end{array}$ & $1.2 \pm 0.02 \mathrm{a}$ & $1.1 \pm 0.03 \mathrm{a}$ & $1.0 \pm 0.01 \mathrm{~b}$ \\
$\begin{array}{l}\text { Densidad real } \\
\left(\mathrm{kg} / \mathrm{m}^{3}\right)\end{array}$ & $2.3 \pm 0.1$ & $2.3 \pm 0.1$ & $2.2 \pm 0.1$ \\
Porosidad (\%) & $51.0 \pm 0.5 \mathrm{a}$ & $50.5 \pm 0.9 \mathrm{a}$ & $53.2 \pm 0.8 \mathrm{~b}$ \\
Materia orgánica (\%) & $2.5 \pm 1.0 \mathrm{a}$ & $2.4 \pm 0.6 \mathrm{a}$ & $3.9 \pm 0.1 \mathrm{~b}$ \\
pH & $7.5 \pm 0.1 \mathrm{a}$ & $7.8 \pm 0.2 \mathrm{a}$ & $7.1 \pm 0.1 \mathrm{~b}$
\end{tabular}

$p<0.0001)$. El bosque tuvo la menor proporción de claros en el dosel y claros basales (Figura 1E-F). La cobertura del suelo desnudo $\left(F_{2,24}=5.9, p<0.008\right)$ fue mayor en la agricultura/
A)

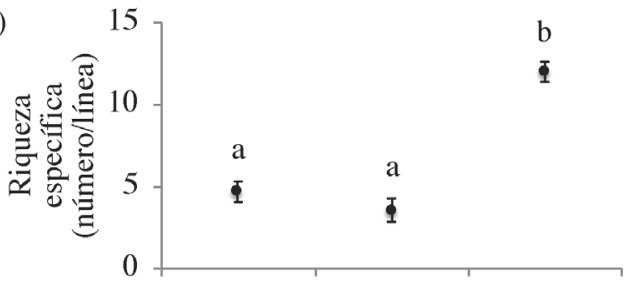

C)

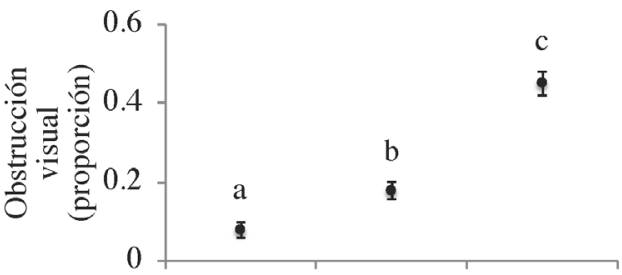

E)

-50-100 cm $\square 101-500 \mathrm{~cm} \square 501-1000 \mathrm{~cm} \backsim>1000 \mathrm{~cm}$



B)

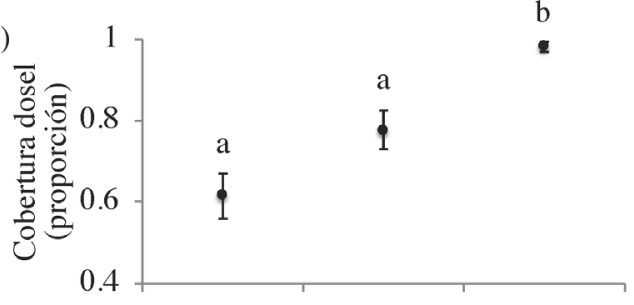

D)
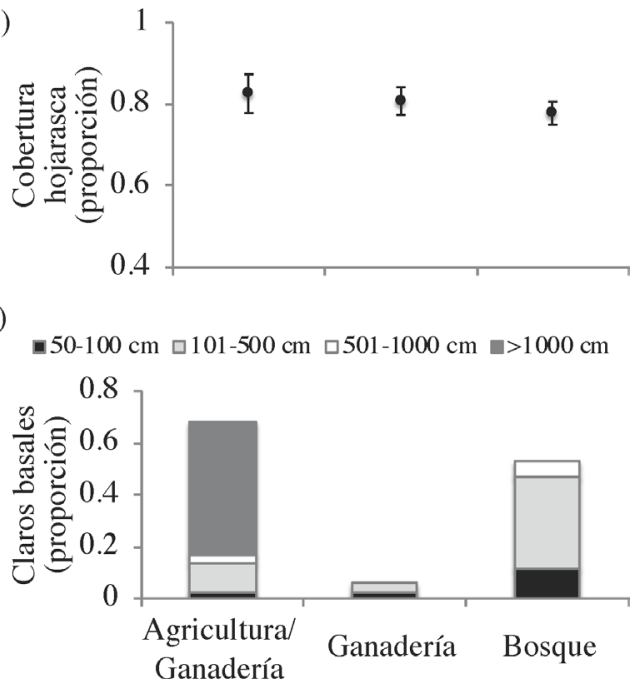

Figura 1. Media ( \pm error estándar) de seis indicadores ecológicos en sitios con distinto manejo. A. riqueza específica de plantas. B. cobertura del dosel. C. obstrucción visual. D. cobertura de hojarasca. E. claros en el dosel. F. claros basales. Para cada indicador, las letras señalan diferencias significativas en el manejo $(p<0.05)$. 
ganadería y ganadería que en el bosque. Sin embargo, la estabilidad del suelo $\left(F_{2,69}=2.6, p<0.08\right)$ fue similar en los tres tipos de manejo (Figura 2A-B). La compactación $\left(F_{2,18}=6.5\right.$, $p<0.008)$ y el tiempo de infiltración $\left(F_{2,18}=5.2, p<0.017\right)$ tuvieron valores altos en la agricultura/ganadería y bajos en el bosque. Los valores en la ganadería fueron intermedios y no difirieron significativamente de la agricultura/ganadería y el bosque (Figura 2C-D).

\section{Discusión}

La agricultura/ganadería y ganadería tuvieron menor estructura y funcionamiento que el bosque, de acuerdo con nuestra hipótesis. Aunque los indicadores ecológicos sólo fueron medidos una vez en el año, nuestros resultados muestran claramente la estructura y el funcionamiento de ambos tipos de manejo, debido a que las mediciones fueron hechas en la temporada de sequía cuando las condiciones ambientales son más estresantes.

Los sitios con agricultura/ganadería y ganadería están en laderas de cerros y tienen suelos con propiedades físicas y químicas relativamente similares. Los sitios con agricultura/ ganadería tuvieron pocas especies de plantas y baja cobertura de dosel. La obstrucción visual fue baja y los claros basales fueron grandes. Además, los suelos tuvieron alta compactación y baja infiltración. Estos resultados son similares a los reportados para otros sitios de bosque tropical seco que han sido transformados para la agricultura de roza, tumba y quema. Los sitios con agricultura de roza, tumba y quema tienen menor diversidad y biomasa de plantas que los sitios con bosque (Miller 1999, Kauffman et al. 2003, DupuyRada et al. 2012). Asimismo, el suelo de estos sitios tiene mayor densidad aparente y menor porosidad total (Giardina et al. 2000, Eaton \& Lawrence 2009, Martínez-Trinidad et al. 2008).

En comparación con la agricultura/ganadería, los sitios con ganadería también tuvieron pocas especies de plantas. Sin embargo, la cobertura del dosel y la obstrucción visual fueron mayores. La proporción de claros basales fue menor. Además, el suelo tuvo menor compactación y mayor infiltración. Otros trabajos realizados en regiones en donde el bosque tropical ha sido transformado a pastizales también han encontrado que éstos tienen baja riqueza específica, cobertura vegetal y biomasa aérea (Kauffman et al. 2003, Arriaga 2006). Además, los suelos tienen mayor densidad aparente y menor porosidad total (García-Oliva et al. 1994, 2006b, Martínez-Trinidad et al. 2008, Sandoval-Pérez et al. 2009).

Aunque las diferencias en la estructura y funcionamiento muestran una tendencia a que la agricultura/ganadería tiene malas condiciones de vegetación y suelo en comparación con la ganadería, no se encontraron diferencias significativas en la mayoría de los indicadores ecológicos. Estos resultados sugieren que la agricultura/ganadería y la ganadería tienen un impacto relativamente similar sobre el bosque tropical. Ambos tipos de manejo simplifican la composición y estructura de la vegetación debido a la remoción de las plantas originales. Además, compactan el suelo y disminuyen la

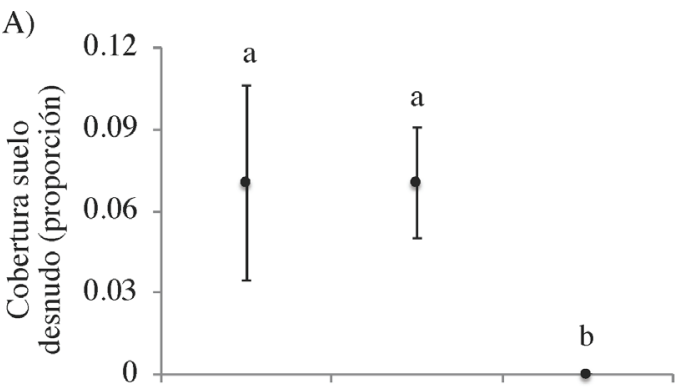

B)


D)

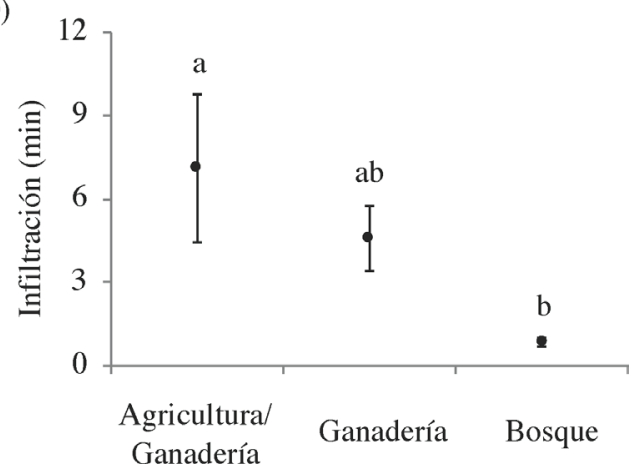

Figura 2. Media ( \pm error estándar) de cuatro indicadores ecológicos en sitios con distinto manejo. A. cobertura de suelo desnudo. B. estabilidad del suelo. C. compactación. D. infiltración. Para cada indicador, las letras señalan diferencias significativas en el manejo $(p<0.05)$. 
infiltración debido a la presencia del ganado. La densidad y el tiempo de permanencia del ganado son diferentes en la agricultura/ganadería (10 cabezas/ha, 7 meses) y ganadería (6 cabezas/ha, 3 meses). Sin embargo, en ambos tipos de manejo, el número de cabezas de ganado es mayor que el índice de agostadero recomendado para Guerrero (6.15 ha/ animal; SEMARNAT 2018), por lo que el manejo del ganado en la agricultura/ganadería y ganadería es inadecuado y podría ocasionar problemas de sobrepastoreo. Por último, la cobertura de la hojarasca y la estabilidad del suelo en ambos tipos de manejo también fueron similares. MartínezTrinidad et al. (2008) tampoco encontraron diferencias en la estabilidad de los agregados del suelo entre un pastizal y un pastizal cultivado en Jalisco.

Las prácticas agrícolas en Zirándaro, Guerrero, difieren de las prácticas agrícolas en Jalisco y Yucatán, por lo que su impacto ecológico sobre el bosque podría ser distinto. En Zirándaro, los restos de plantas no son quemados después de la roza por lo que podrían contribuir a retener agua y nutrientes en el suelo. Además, la ausencia de quema de los restos de plantas disminuiría la probabilidad de incendios, la destrucción del banco de semillas y la pérdida de materia orgánica del suelo. Por el contrario, en Jalisco y Yucatán, los restos vegetales derivados de la roza son esparcidos en la milpa y quemados para liberar nutrientes en la superficie del suelo. No obstante, la mayoría de estos nutrientes se pierden del suelo debido a un incremento de la erosión hídrica y eólica (Challenger 1998, García-Oliva \& Maass 1998, Giardina et al. 2000). Estas diferencias sugieren que la agricultura/ ganadería en Zirándaro podría tener un impacto ecológico menor que la roza, tumba y quema en Jalisco y Yucatán. Sin embargo, para probar esta hipótesis es necesario medir los almacenes y dinámica de algunos nutrientes del suelo como el carbono, nitrógeno y fósforo en Zirándaro.

En conclusión, la agricultura/ganadería y ganadería disminuyen la estructura y funcionamiento del bosque tropical seco de Zirándaro, Guerrero. Ambos tipos de manejo tienen un impacto ecológico relativamente similar sobre el bosque. Sin embargo, el impacto de la agricultura/ganadería en Zirándaro podría ser menor que el impacto de la agricultura de roza, tumba y quema en otras regiones de bosque tropical seco de nuestro país.

\section{Agradecimientos}

Este artículo está dedicado a nuestro amigo Agustín FuentesHernández, quien desafortunadamente falleció antes de poder concluir el estudio. El estudio fue apoyado por el Programa de Apoyo a los Profesores de Carrera para promover Grupos de Investigación (PAPCA), Facultad de Estudios Superiores Iztacala, Universidad Nacional Autónoma de México a través del subsidio FESI-DIP-PAPCA-2014-25.

\section{Literatura citada}

Arriaga L. 2006. La península de Baja California: diversidad, conservación y manejo de sus recursos vegetales. En: Oyama K, Castillo A. eds. Manejo, Conservación y Restauración de
Recursos Naturales en México. México: Siglo XXI EditoresUNAM, 64-84. ISBN: 9789682326493

Challenger A. 1998. Utilización y conservación de los ecosistemas terrestres de México. Pasado, presente y futuro. México: CONABIO. ISBN: 970-9000-02-0

Dupuy-Rada J, Hernández-Stefanoni JL, Hernández-Juárez R, Tun-Dzul F, May-Pat F. 2012. Efectos del cambio de uso del suelo en la biomasa y diversidad de plantas leñosas en un paisaje de bosque tropical seco en Yucatán. Investigación Ambiental 4: 130-140.

Eaton JM, Lawrence D. 2009. Loss of carbon sequestration potential after several decades of shifting cultivation in the Southern Yucatán. Forest Ecology and Management 258: 949-958. DOI: https://doi.org/10.1016/j.foreco.2008.10.019

García E. 2004. Modificaciones al Sistema de Clasificación Climática de Köppen. México DF: Serie libros. Instituto de Geografía-UNAM. ISBN: 970-32-1010-4

García-Oliva F, Casar I, Morales P, Maass JM. 1994. Forest-to-pasture conversion influences on soil organic carbon dynamics in a tropical deciduous forest. Oecologia 99: 392-396. https://doi.org/10.1007/BF00627754

García-Oliva F, Maass JM. 1998. Efecto de la transformación de la selva a pradera sobre la dinámica de los nutrientes en un ecosistema tropical estacional en México. Boletín de la Sociedad Botánica de México 62: 39-48.

DOI: https://doi.org/10.17129/botsci.1549

García-Oliva F, Maass JM, Jaramillo VJ, Martínez-Yrízar A, Pérez-Jiménez A, Sarukhán J. 2006a. El enfoque ecosistémico y su aplicación en problemas de manejo y restauración ecológica: el caso de la selva baja caducifolia en Chamela, Jalisco. En: Oyama K, Castillo A. eds. Manejo, Conservación y Restauración de Recursos Naturales en México. México: Siglo XXI Editores-UNAM, 111-126. ISSN: 9789682326493

García-Oliva F, Gallardo-Lancho JF, Montaño NM, Islas P. 2006b. Soil carbon and nitrogen dynamics followed by a forest-to-pasture conversion in western Mexico. Agroforestry Systems 66: 93-100. https://doi.org/10.1007/s10457-005-2917-z

Giardina CP, Sanford RL, Døckersmith IC, Jaramillo VJ. 2000. The effects of slash and burning on ecosystem nutrients during the land preparation phase of shifting cultivation. Plant and Soil 220: $247-260$. https://doi.org/10.1023/A:1004741125636

INEGI.2001. Anuario Estadístico del Estado de Guerrero. México: Instituto Nacional de Estadística, Geografía e Informática.

Hernández-Vargas G, Sánchez-Velásquez LR, Carmona-Valdovinos TF, Pineda-López MR, Cuevas-Guzmán R. 2000. Efecto de la ganadería extensiva sobre la regeneración arbórea de los bosques de la Sierra de Manantlán. Madera y Bosques 6: 13-28. DOI: http://dx.doi.org/10.21829/myb.2000.621332

Herrick JE, van Zee JW, Havstad KM, Burkett LM, Whitford WG. 2009a. Monitoring Manual for Grassland, Shrubland and Savanna Ecosystems. Volume I: Quick Start. USDA-ARS Jornada Experimental Range. Arizona: The University of Arizona Press. ISBN 0-9755552-0-0

Herrick JE, van Zee JW, Havstad KM, Burkett LM, Whitford WG. 2009b. Monitoring Manual for Grassland, Shrubland and Savanna Ecosystems. Volume II: Design, Supplemen- 
tary Methods and Interpretation. USDA-ARS Jornada Experimental Range. Arizona: The University of Arizona Press. ISBN 0-9755552-0-0

Herrick JE, van Zee JW, McCord SE, Courtright EM, Karl JW, Burkett LM. 2017. Monitoring Manual for Grassland, Shrubland and Savanna Ecosystems. Volume I: Core Methods. USDA-ARS Jornada Experimental Range. Arizona: The University of Arizona Press. ISBN 0-9755552-0-0

Kauffman JB, Steele MD, Cummings DL, Jaramillo VJ. 2003. Biomass dynamics associated with deforestation, fire, and conversion to cattle pasture in a Mexican tropical dry forest. Forest Ecology and Manangement 176: 1-12.

DOI: https://doi.org/10.1016/S0378-1127(02)00227-X

Martínez-Trinidad S, Cotler H, Etchevers-Barra JD, OrdazChaparro VM, León-González F. 2008. Efecto del manejo en la agregación del suelo en un ecosistema tropical seco. Terra Latinoamericana 26: 299-307.

Meave JA, Romero-Romero MA, Salas-Morales SH, PérezGarcía EA, Gallardo-Cruz JA. 2012. Diversidad, amenazas y oportunidades para la conservación del bosque tropical caducifolio en el estado de Oaxaca, México. Ecosistemas 21: 85-100.

Miller PM. 1999. Effects of deforestation on seed bank in a tropical deciduous forest of western Mexico. Journal of Tropical Ecology 15: 179-188.

DOI: http://dx.doi.org/10.1017/s0266467499000747

Morales-Romero D, Molina-Freaner F. 2016. Conversion of thornscrub to buffelgrass pasture in northwestern Mexico: Microclimatic consequences. Journal of Arid Environments 125: $110-115$.

DOI: https://doi.org/10.1016/j.jaridenv.2015.10.012

Muñoz ID, Soler A, López, F, Hernández M. 2013. Edafología. Manual de Métodos de Análisis de Suelos. México: Facultad de Estudios Superiores Iztacala, UNAM.

Pellant M, Shaver P, Pyke DA, Herrick JE. 2005. Interpreting Indicators of Rangeland Health, Version 4. Technical Reference 1734-6. Denver: U.S. Department of Interior, Bureau of Land Management, National Science and Technology Center.

Pyke DA, Herrick JE, Shaver P, Pellant M. 2002. Rangeland health attributes and indicators for qualitative assessment. Journal of Range Management 55: 584-597. DOI: http://dx.doi.org/10.2307/4004002

Sandoval-Pérez AL, Gavito ME, García-Oliva F, Jaramillo, VJ. 2009. Carbon, nitrogen, phosphorus and enzymatic activity under different land uses in a tropical, dry ecosystem. Soil Use and Management 25: 419-426. DOI: https://doi.org/10.1111/j.1475-2743.2009.00234.x

SAS. 2012. SAS Institute Inc. Using JMP 10. Cary: SAS Institute.

SEMARNAT [Secretaría de Medio Ambiente y Recursos Naturales]. 2018. Coeficientes de agostadero por entidad. <http:// dgeiawf.semarnat.gob.mx $>$ (accessed november 29, 2018).

Editor de sección: Enrique Jurado

Contribución de los autores: AFH y MMO participaron en el trabajo de campo, LRC participó en el trabajo de campo y escritura del manuscrito, ASA participó en los análisis de suelo, DMI participó en los análisis de suelo y revisión del manuscrito, HGÁ participó en el trabajo de campo, análisis de datos y escritura del manuscrito. 